\title{
Compétences et apprentissages de l'anglais de spécialité
}

Exemples de cursus professionnalisés en publicité et en documentation d'entreprise à l'IUT Michel de Montaigne - Bordeaux 3

\section{Michel Coupal}

\section{(2) OpenEdition}

Journals

Édition électronique

URL : http://journals.openedition.org/asp/1518

DOI : 10.4000/asp.1518

ISBN : 978-2-8218-0390-9

ISSN : 2108-6354

\section{Éditeur}

Groupe d'étude et de recherche en anglais de spécialité

\section{Édition imprimée}

Date de publication : 30 décembre 2002

Pagination : 109-120

ISSN : 1246-8185

Référence électronique

Michel Coupal, « Compétences et apprentissages de l'anglais de spécialité », ASp [En ligne],

37-38 | 2002, mis en ligne le 25 juillet 2010, consulté le 01 mai 2019. URL : http://

journals.openedition.org/asp/1518; DOI : 10.4000/asp.1518

Ce document a été généré automatiquement le 1 mai 2019.

Tous droits réservés 


\section{Compétences et apprentissages de l'anglais de spécialité}

Exemples de cursus professionnalisés en publicité et en documentation d'entreprise à l'IUT Michel de Montaigne - Bordeaux 3

\section{Michel Coupal}

\section{Introduction}

1 Il s'agit ici de présenter un bref compte rendu de nos activités d'enseignement/ apprentissage de l'anglais à des étudiants de deuxième année dans les filières Publicité et Information et documentation d'entreprise à l'IUT Michel de Montaigne - Bordeaux 3. Notre perspective ne s'inscrit pas dans une discussion théorique des problèmes posés par l'enseignement de langue étrangère à des publics spécifiques. Nous nous situons dans une perspective de pratique pédagogique exemplifiée par des documents joints en annexe, dont nous exposerons les étapes de réalisation. Ces travaux, cependant, s'inscrivent dans le cadre de présupposés théoriques sur l'apprentissage qui se feront jour, nous l'espérons, dans le déroulement de notre exposé. Néanmoins, il ne nous semble pas inutile d'en présenter brièvement les orientations fondamentales.

2 Nous référant à la présentation de Denis Lehmann (1993) nous remettons en cause une démarche systématique fondée sur une analyse des besoins a priori, qu'ils soient de nature linguistique ou situationnelle. Nous focalisons notre approche sur l'organisation d'activités à accomplir par les étudiants plutôt que sur l'acquisition de structures linguistiques prédéterminées en fonction de besoins de communication. La communication, par sa nature même, incluant un interlocuteur, nécessite un ajustement constant des participants à l'échange et ne présente donc pas un caractère de prévisibilité systématique (cf. la recommandation publicitaire).

D'autre part nous disposions d'un nombre d'heures restreint qui ne s'accommoderait pas d'une recherche naïve d'exhaustivité linguistique ou situationnelle. Nous postulons que l'utilisation de la langue dans ces activités facilite son acquisition de manière plus efficace 
que des manipulations sur le système lui-même. Cette position n'est pas aussi radicale qu'il n'y paraît puisqu'elle n'exclut pas le travail sur la langue dans le cadre de ces activités, comme en témoigne la suite de la présentation. Il serait vain de croire qu'on puisse en faire l'économie.

4 Les modifications portent sur la centration de l'enseignement/apprentissage. L'imprévisibilité de la communication et le facteur temps nous ont conduit à privilégier une centration sur les processus d'apprentissage et l'acquisition de compétences. Nous visons à développer chez l'apprenant ses capacités d'analyse, la mise en œuvre de stratégies qui lui permettent de gérer les situations de communication nouvelles auxquelles il devra faire face en langue étrangère. À terme nous visons à lui fournir les moyens d'aborder les problèmes linguistiques et culturels afférents à ses activités personnelles et à lui conférer ainsi son autonomie.

5 L'ambition est grande, les problèmes nombreux et les résultats exposés ci-dessous sont livrés à l'appréciation et aux critiques.

\section{Description des situations d'apprentissage}

6 Les situations que nous allons présenter s'inscrivent dans le cadre d'une préparation à l'utilisation de l'anglais dans une situation professionnelle ${ }^{1}$ et relèvent de tâches précises: la production d'une recommandation publicitaire en langue anglaise; la réalisation d'un "sitoscope $"^{2}$ en anglais. Dans les deux cas, les activités sont très codifiées et donnent lieu à l'élaboration d'un produit formaté selon les règles de la profession. Ces productions sont la transposition en langue anglaise de prestations orales ou écrites connues de l'étudiant dans le cadre de son apprentissage professionnel. Ces tâches impliquent la mise en œuvre de stratégies d'apprentissage qu'il s'agit de présenter en décrivant les spécificités de chacune des productions et en dégageant les compétences à acquérir et les moyens mis en œuvre pour atteindre l'objectif d'une réalisation de qualité professionnelle. Nous présenterons en deux temps les produits à réaliser car les situations d'apprentissage ne sont pas superposables.

\subsection{La recommandation publicitaire}

7 Présentée devant un jury de professionnels anglophones de la publicité, la recommandation publicitaire est un travail collectif constitué d'une présentation orale avec un support écrit de propositions visant à résoudre un problème de communication publicitaire ou de stratégie de commercialisation d'un produit ou service. Cette session d'une semaine $(20 \mathrm{~h})$ conclut un enseignement hebdomadaire d'anglais $(20 \mathrm{~h})$ de la publicité au cours duquel les étudiants se familiarisent avec le vocabulaire et la culture publicitaires dans les pays anglo-saxons. Il est important de souligner ici que le problème publicitaire est fondé sur un produit ou service réel qui a déjà fait l'objet d'une présentation en langue maternelle. Le sujet en anglais est la transposition dans un cadre européen d'une recommandation pour le marché français présentée deux semaines auparavant. La mise en situation est celle d'une présentation à un client anglophone des études stratégiques et des recommandations publicitaires qui en découlent. Le sujet ${ }^{3} \mathrm{du}^{\mathrm{a}}$ cas à l'étude est donné une semaine auparavant. La prestation de groupe comporte donc différentes composantes qui nécessitent la mise en œuvre de compétences diverses.

Synthèse des informations connues sur le sujet 
8 À partir du brief donné par les professionnels pour le cas en français, il s'agit de déterminer les contours du problème à résoudre et d'extraire les informations nécessaires à la résolution du problème dans un contexte européen. Les données ne sont pas toujours complètes ou adaptées, les étudiants doivent donc trouver les informations complémentaires qu'ils jugent nécessaires. Le fait que le produit et le marché français dans lequel il s'insère soient connus facilite la détermination des lacunes en matière d'information et permet aux étudiants de cibler leurs recherches.

Recherche d'information complémentaire

9 À partir de documents en anglais fournis par l'enseignant et de recherches personnelles dans des revues spécialisées ou sur l'Internet, les étudiants complètent et adaptent l'information qui leur est nécessaire pour les étapes suivantes.

Élaboration d'une stratégie publicitaire

10 Cette étape, réalisée en français hors de la relation enseignante, est primordiale à la qualité de la prestation finale. Il est illusoire de penser que la réflexion publicitaire puisse se faire dans une autre langue que la langue maternelle. Mais il est aussi essentiel pour l'enseignant de vérifier que les productions issues de cette réflexion professionnelle soit en cohérence avec la langue et la culture de destination.

Réalisation

11 La recommandation comporte trois éléments: la production d'un document écrit présentant le produit, le marché et la concurrence, les habitudes de consommation, la stratégie retenue et les moyens de sa mise en œuvre; la fabrication de matériels publicitaires media et hors media; la préparation de la présentation orale avec un support PowerPoint.

Présentation de la recommandation

12 Le groupe d'étudiants se partage la prestation orale en fonction de leurs intérêts et compétences linguistiques. Ils répondent aux questions et réagissent aux remarques du jury sur la partie de la recommandation qui les concernent.

\subsubsection{Compétences nécessaires}

13 Outre les compétences en matière publicitaire sur lesquelles se fondent essentiellement cet exercice, les étudiants développent des compétences linguistiques et culturelles nécessaires à la production jugée professionnellement :

1. maîtrise du vocabulaire technique : jargon et culture publicitaire ;

2. compréhension : langue et culture étrangère ;

3. expression écrite: adaptation au style «efficace » et direct qui caractérise la langue publicitaire des pays anglophones ;

4. expression orale : transcription à l'oral de cette concision publicitaire ;

5. traitement de l'information;

6. identification des besoins.

\subsubsection{Moyens de la mise en œuvre}

14 Compréhension: des exercices d'extraction d'information permettent aux étudiants de repérer l'information pertinente. 
Lexique : le jargon publicitaire est acquis au fur et à mesure d'exercices préparatoires pendant les cours réguliers. Parallèlement, travail sur les temps du verbe et les qualificatifs.

Stylistique : les caractéristiques du style publicitaire en anglais sont illustrées par des exemples et mises en évidence par des exercices au cours des séances hebdomadaires.

Expression orale : des séances de coaching sont prévues afin de corriger l'expression et d'affiner le caractère synthétique de la prestation devant un client. Il s'agit d'aller à l'essentiel, de prévoir les enchaînements afin de rendre fluide et le moins scolaire possible la production orale du groupe.

\subsubsection{Tâches préparatoires}

8 L'objectif de la préparation hebdomadaire est de familiariser les étudiants avec les stratégies qu'ils devront mettre en œuvre. Des exemples de recommandations professionnelles permettent aux étudiants de se familiariser avec les différences qui existent entre les cultures publicitaires française et anglo-saxonne.

À partir d'un texte sur le lancement d'un produit, les étudiants identifient et structurent les diverses rubriques que comporte une recommandation en anglais. Ces activités sont définies sur un document remis aux étudiants et donnent lieu à une discussion sur le champ sémantique que recouvrent les termes. Nous évitons à ce moment de l'acquisition des connaissances de développer la synonymie qui caractérise l'expression publicitaire afin de se concentrer sur un formatage des concepts plutôt que sur un développement du lexique.

20 Le visionnement d'un vidéogramme sur les habitudes de consommation des Britanniques permet la prise en compte des différences comportementales et professionnelles entre deux cultures. Il s'agit ici d'une recherche de pertinence par l'extraction du document des caractéristiques du comportement consumériste, une structuration en catégories qui ne sont pas les mêmes qu'en France et une identification des éléments jugés les plus représentatifs. Les étudiants concilient ainsi acquisition de référents cultures et développement d'un vocabulaire descriptif concis et adapté à la tâche publicitaire.

La description d'images publicitaires, la rédaction de publicités radiophoniques permettent l'acquisition du vocabulaire de la description et renforce la maîtrise du format retenu pour la prestation orale. Les étudiants développent des compétences de synthèse et d'efficacité de la description tout en acquérant le métalangage de la communication publicitaire.

22 Un exercice sur les slogans et les accroches en publicité confronte les jeunes publicitaires aux différences stylistiques et culturelles du message publicitaire en leur faisant prendre conscience du résultat, inacceptable ou souvent incompréhensible, d'une traduction mot à mot $^{4}$. La prise de conscience qu'un message s'adresse avant tout à une culture conduit à la réalisation de la nécessaire adaptation d'un concept stratégique conçu en français à une réalité linguistique, temporelle et culturelle distincte.

3 Un exercice de présentation orale d'une recommandation simplifiée permet la mise en œuvre des techniques orales et écrites acquises pendant les séances hebdomadaires. Si, contrairement à la recommandation finale, les étudiants ont droit à une fiche, qu'ils se contentent souvent de lire, cet exercice permet d'appréhender la difficulté de la tâche et de la nécessaire concision d'une prestation orale. Les remarques à chaud de l'enseignant 
et l'autoévaluation par le visionnement de l'enregistrement vidéo effectué à cette occasion contribuent à renforcer la prise de conscience que l'expression en anglais ne peut être qu'une simple traduction d'un texte conçu et rédigé en français.

Des exercices autour des contraintes législatives ou formelles contribuent à l'acquisition d'une culture publicitaire par la prise de conscience que l'on ne peut pas toujours transposer en anglais un simple fac-simile d'une publicité française. L'incitation à la lecture de textes professionnels et le visionnement de publicités en langue étrangère font mesurer, une fois dépassés les premiers commentaires sur le caractère exotique ou ridicule de la culture étrangère, l'importance de bien connaître la culture à laquelle est adressée le message publicitaire.

\subsection{Le sitoscope}

25 Le produit documentaire qui est l'aboutissement du cours d'anglais de spécialité en Information et documentation d'entreprise est, contrairement à la recommandation publicitaire, un travail individuel, parfois en groupe de deux selon la disponibilité du matériel informatique. La production de ces étudiants de deuxième année est sanctionnée professionnellement par la mise en ligne ou non de leur page Web. Comme nous l'avons indiqué, il s'agit ici aussi d'une réalisation formatée qui répond aux exigences de la profession en matière de justesse d'analyse et de clarté de communication. Le sitoscope, que nous pouvons décrire comme un mini portail de liens thématiquement structurés et commentés, est un produit protéiforme dont la réalisation n'est pas réservée uniquement aux documentalistes. Mais le format proposé aux étudiants réunit les contraintes d'une réalisation documentaire professionnelle combinant les exigences de pertinence, de lisibilité, d'actualité et d'exhaustivité. Sans oublier la notion de valeur ajoutée par le documentaliste à toute présentation d'un produit d'information, c'est-à-dire qu'il ne s'agit pas d'une simple collecte, mais d'une structuration raisonnée et une approche guidée pour un public déterminé à des informations disponibles par ailleurs.

Comme pour la publicité, le produit final est la transposition en anglais d'un ensemble de compétences professionnelles acquises dans le cursus de la formation. Les exigences linguistiques viennent renforcer les acquis de la formation et, par conséquent, la langue étrangère ne peut être abstraite des fonctionnements spécifiques à la profession. Il s'agit d'adapter en langue anglaise une pratique connue dans une réalisation qui est toutefois nouvelle. En effet, le sitoscope en anglais est la seule réalisation de ce genre. Les étudiants ont l'occasion de créer plusieurs produits documentaires papiers ou électroniques (répertoires, bibliographies, bases de données ou mémoires) au cours de leur formation, mais le bookmark thématique est réalisé en parallèle avec le cours de langages du Web dans lequel ils apprennent à réaliser de pages Web. Le cours d'anglais s'appuie donc sur des techniques documentaires acquises par ailleurs: analyse des besoins, recherche sur l'Internet, évaluation de sites Web, construction de classements hiérarchiques, indexation, résumé, etc. Il est aussi l'occasion de réitérer ces pratiques qui, contrairement à l'environnement publicitaire, ne comportent pas de différences culturelles marquées.

27 Les étapes de réalisation du produit final sont les suivantes 5 .

Analyse des besoins

28 La tâche est présentée comme la création d'une page Web utile pour les étudiants en Information et documentation d'entreprise. Ils puisent donc dans leur expérience de 
recherche sur l'Internet pour choisir le thème de leur produit. La validation de ce choix passe par l'examen doublé d'une évaluation des réalisations des années précédentes.

Recherche documentaire

Une fois le sujet déterminé et validé, la recherche des sites pertinents permettra de constituer un corpus qui constituera la sélection pertinente à l'objet à réaliser. Les techniques de recherches étant les mêmes, il s'agira ici de développer les capacités de repérages de l'information afin d'améliorer l'équation de recherche. Il est aussi nécessaire de faire prendre conscience qu'une réalité ne s'exprime pas d'une seule manière et qu'existent des différences linguistiques, culturelles, discursives parmi toutes les informations disponibles en langue anglaise. Une connaissance préalable des divers outils de recherche et de leurs spécificités est nécessaire afin d'acquérir des réflexes professionnels qui distingueront le « cyberdocumentaliste » du simple « surfeur ».

Exploitation des résultats

Les sites qui ont été retrouvés ${ }^{6}$ font alors l'objet d'une évaluation en fonction de leur pertinence par rapport au produit final. Seront retenus les sites qui satisfont aux autres critères professionnels déjà mentionnés.

Hiérarchisation des concepts

31 L'examen des sites trouvés conduit à la rédaction d'un menu dans lequel l'étudiant mettra en œuvre une hiérarchisation des contenus en fonction du sujet de son site. Cette construction de relations hiérarchiques peut-être géographique, typologique, génériquespécifique, etc. Il faut éviter la simple liste alphabétique qui est contreproductive en matière d'analyse des contenus.

Rédaction des commentaires

32 Afin d'éviter le couper/coller si fréquent dans ce genre d'exercice, l'étudiant doit non seulement rédiger une courte description du contenu informatif du site mais il doit $\mathrm{y}$ ajouter une justification de l'utilité documentaire de ses choix pour le public visé, c'est-àdire un étudiant comme lui.

Mise en forme en langage Web

33 La création de la page étant techniquement prenante, elle se fait hors du cours d'anglais mais l'enseignant contrôle à plusieurs reprises la rédaction afin de signaler aux étudiants sans les corriger les fautes et les maladresses pouvant nuire à la compréhension de son produit.

\subsubsection{Compétences à développer} s'agit de produire un document qui puisse être compris et utilisé par un étudiant en Science de l'information en France ou à l'étranger. familiariser avec le vocabulaire particulier du sujet retenu. 


\subsubsection{Tâches préparatoires}

39 Afin d'assurer une certaine qualité à ce travail presque autonome des étudiants, les exercices suivants viennent compléter la formation qu'ils reçoivent dans leurs enseignements théoriques et technologiques en français. La plupart de ces tâches donnent lieu à une communication par courriel avec l'enseignant et à un retour d'information ou une correction de la part de ce dernier.

En faisant appel à une référence déjà existante, c'est-à-dire en évaluant les sites des promotions précédentes, les étudiants se familiarisent avec le format du produit final et de construisent le schème des attentes professionnelles de l'enseignant. À partir de grilles d'évaluation trouvées par les étudiants sur l'Internet, ceux-ci sont amenés à repérer les critères professionnels d'un produit documentaire.

41 La lecture des commentaires leur permet de mesurer le degré d'utilité annoncé vis-à-vis de leurs propres besoins documentaires. Ils constatent ainsi qu'une formulation simplifiée est plus parlante que le travail d'un funambule du couper/coller.

42 Après des recherches exploratoires, la sélection du sujet donne lieu à l'envoi par courrier électronique d'une proposition de titre et de courte présentation des raisons de ce choix. En contextualisant sous forme concise son choix et en justifiant de l'utilité de celui-ci, l'étudiant construit le titre et l'introduction de son site Web.

43 Une fois le thème validé et le champ sémantique identifié, l'étudiant procède à une exploration plus rigoureuse qui le mène à construire une stratégie de recherche constituée d'une équation, c'est-à-dire la sélection des termes ou phrases qui feront l'objet de la recherche, et du choix des outils. Les diverses équations sont ensuite l'objet d'une discussion de groupe (en français) qui permet à l'enseignant de revenir sur les notions de champs sémantiques, de différences culturelles et de fonctionnalités des outils de recherche qui pourraient avoir échappé à certains. Ce travail sur le lexique se fait par un aller-retour constant entre la structuration initiale et la réalité de l'information retrouvée. Il est important de s'arrêter ici sur le travail préparatoire de la recherche documentaire sur l'Internet ou sur tout autre support.

La première question à se poser est " ai-je bien compris la demande d'information?».

44 Ici les besoins sont supposés connus de l'étudiant, puisqu'il s'agit de ses propres besoins. Mais contrairement à une situation professionnelle dans laquelle la demande provient d'un tiers et fait l'objet d'un aller-retour permanent, il doit avoir recours à l'autoévaluation. La mesure de l'efficacité de sa prestation ne sera possible qu'en la confrontant à d'autres utilisateurs.

"Qui est susceptible de produire l'information recherchée?»

45 Les étudiants doivent s'appuyer ici sur une connaissance de la culture cible: sources d'information officielles et autres, organismes universitaires et de recherche, activité économique, producteurs d'information spécialisée, etc.

«Quels termes retenir?»

46 L'exploration du champ sémantique dépend de la détermination préalable des concepts qui définissent l'objet recherché. En dégageant les idées maîtresses que doivent contenir les documents trouvés. Les étudiants construisent ensuite des listes hiérarchisées et/ou synonymiques de vocables et d'expression utilisés dans le jargon professionnel du sujet 
choisi. La richesse de cette sélection dépendra de la capacité de l'étudiant à extraire des documents l'information utile.

"Quelle stratégie de recherche adopter?»

Il s'agit ici d'anticiper les réponses qui seront obtenues avec l'équation de recherche. Il faut prévoir des équations permettant d'élargir ou d'affiner les champs de recherche ou de faire appel à des outils complémentaires.

«Est-ce l'information recherchée?»

48 La réponse à cette question dépendra de l'intérêt de l'étudiant pour son sujet et de son imprégnation de ses manifestations linguistiques et culturelles. C'est par un travail allerretour évaluatif permanent que l'étudiant construira un produit satisfaisant aux normes professionnelles. Dans sa version finale, le produit est ensuite mis en ligne sur un serveur école afin que l'étudiant puisse en vérifier le contenu et la forme. Une première autoévaluation est réalisée en demandant aux étudiants d'examiner et de commenter les productions des autres membres de la classe. L'évaluation par l'enseignant se fait sous forme de fiche reprenant les notions professionnelles d'adéquation à l'objectif annoncé d'actualité, de lisibilité et d'exhaustivité, ainsi qu'une appréciation de la qualité de la langue mise en œuvre : correction, adéquation et pertinence des commentaires.

\section{Conclusion}

49 Ces réalisations ont en commun la sanction de la réalité professionnelle. Cette inscription dans une pratique professionnelle conditionne l'apprentissage en langue anglaise en imposant aux étudiants de ne jamais s'abstraire de leur langue maternelle ou de la construction des connaissances à partir de leur culture professionnelle de départ. Il nous est donc impossible de focaliser sur la seule langue à acquérir.

Elles se fondent aussi toutes deux sur la motivation générée par ce même souci de professionnalisme issu de la sanction qui n'est pas une simple notation mais une gratification inscrite dans un cursus professionnel. La créativité du publiciste est récompensée par les observations d'un professionnel respecté et les capacités d'analyse et de synthèse du documentaliste sont sanctionnées par la mise à disposition du monde entier de leur production.

51 Les productions et les stratégies mises en œuvre se distinguent par la manière avec laquelle ces deux professions utilisent le langage. Le fonctionnement métaphorique de la langue publicitaire incite à un recours systématique aux référents culturels français, il est essentiel de faire prendre conscience de l'impossibilité d'avoir recours à la seule traduction. En Documentation, au contraire, le transfert permet de construire une stratégie menant à la découverte du lexique essentiel du champ d'investigation. Sans celui-ci, la recherche d'information et la hiérarchisation des résultats donneront une production sans intérêt.

De même, le recours à des stratégies d'évitement est impossible en Publicité puisqu'il y a nécessité de construire une réflexion stratégique originale et adaptée au problème. En Documentation, les stratégies d'évitement choisies par les étudiants les moins motivés conduisent souvent à des réalisations jugées moins riches et moins pertinentes. 


\section{BIBLIOGRAPHIE}

Lehmann, Denis. 1993. Objectifs spécifiques en langue étrangère. Les programmes en question. Paris : Hachette.

\section{NOTES}

1. Remerciements : Je tiens à mentionner ici mes collègues Pierre Lacrampe et Hubert Cahuzac qui sont à l'initiative de ces productions et qui m'ont permis de développer leur transposition dans le cadre de l'enseignement de l'anglais de spécialité.

2. Nous avons défini le sitoscope comme une collection thématiquement structurée et commentée de liens vers des sites Internet, un bookmark raisonné en quelque sorte.

3. Parmi les sujets proposés au cours des dernières années, nous trouvons des recommandations pour des déodorants, des camemberts, des voitures ou des revues.

4. L'exemple de la traduction du slogan Fly Lufthansa you won't believe your eyes par la phrase "Volez Lufthansa vous n'en reviendrez pas » est à cet effet très éloquent. De même que le lancement raté d'une boisson gazeuse française affublée du nom de Jizz sur le marché américain.

5. Notons que si les indexations et les résumés scientifiques ou techniques en anglais diffèrent grammaticalement ou stylistiquement, les autres compétences professionnelles s'appuient sur les mêmes techniques et méthodes.

6. L'équivalent anglais de l'expression française recherche d'information est en effet information retrieval.

\section{RÉSUMÉS}

L'auteur présente deux situations d'apprentissage de l'anglais de spécialité dans les domaines de la publicité et de la documentation d'entreprise. Il décrit les tâches à accomplir et les compétences nécessaires afin de dégager les stratégies mises en œuvre dans la réalisation de produits professionnels formatés: une recommandation publicitaire et une bibliographie électronique en ligne.

The author presents two examples of project-based learning in EFL with students in Librarianship and Information Science at the Institute of Technology of the University of Bordeaux 3. In professional context, he describes the tasks leading to the making of an advertising recommendation and an online Web bibliography underlining the necessary language and strategic skills. 
INDEX

Mots-clés : anglais de spécialité, apprentissage, compétence, documentation d'entreprise, projet, publicité, tâche

Keywords : advertising, EFL, information science, learning situation, librarianship, skill, strategy, task-based project

\section{AUTEUR}

MICHEL COUPAL 